Revue d'histoire de l'Amérique française

RAS REVUE D.HISTOIRE DE L'AMÉRIQUE FRANÇAISE

\title{
Le commerce compliqué des fourrures canadiennes au début du XVII ${ }^{\mathbf{e}}$ siècle
}

\section{Robert Le Blant}

Volume 26, numéro 1, juin 1972

URI : https://id.erudit.org/iderudit/303151ar

DOI : https://doi.org/10.7202/303151ar

Aller au sommaire du numéro

Éditeur(s)

Institut d'histoire de l'Amérique française

ISSN

0035-2357 (imprimé)

1492-1383 (numérique)

Découvrir la revue

Citer cet article

Le Blant, R. (1972). Le commerce compliqué des fourrures canadiennes au début du XVII ${ }^{\mathrm{e}}$ siècle. Revue d'histoire de l'Amérique française, 26(1), 53-66. https://doi.org/10.7202/303151ar d'utilisation que vous pouvez consulter en ligne.

https://apropos.erudit.org/fr/usagers/politique-dutilisation/ 


\title{
LE COMMERCE COMPLIQUÉ DES FOURRURES CANADIENNES AU DÉBUT DU XVII ${ }^{e}$ SIËCLE
}

\author{
Robert Le Blant \\ Paris, France
}

Les contrats portant sur le commerce des fourrures au début $\mathrm{du} \mathrm{XVII}^{\mathrm{e}}$ siècle nous fournissent des renseignements sur les fluctuations des prix, sur la faveur qu'obtenaient les diverses peaux sur le marché français, sur les formes d'association, sur les litiges qui surgissaient entre associés ou entre groupes de marchands. Nous en offrons ici quelques illustrations.

Les castors étaient encore connus en France, à la fin du $\mathrm{XVI}^{\mathbf{e}}$ siècle, sous le nom de bièvres, nom qui ne s'appliquait pas seulement aux fourrures parisiennes, françaises ou européennes, car le 7 décembre 1586, Mathieu Garnier, pelletier et valet de chambre du roi demeurant rue de la Lingerie à Paris, et Mathieu Soupplet, maître chapelier demeurant rue des Gants, paroisse Saint-Merry, donnèrent procuration à Mathieu Soupplet, dit le jeune, fils du précédent, pour se rendre à Bayonne et fixer avec René Daret, marchand bourgeois de cette ville, le prix des pelleteries provenant du lieu de Terre-Neuve, telles que bièvres, loutres et martres. ${ }^{1}$ Le plus ancien acte connu portant sur le commerce des peaux de castor est celui du 19 mai 1603, attestant un envoi de cette marchandise à Koenigsberg par le marchand rochelais Jean Macain. ${ }^{2}$ Son associé Samuel Georges subissait à la même époque la saisie effectuée à la douane de Paris sur requête des maîtres et gardes de la pelleterie de cette ville de trois balles de castors propres à faire les chapeaux. ${ }^{3}$ Les gardes agissaient sur la demande de l'un des leurs, Henri Honton représentant d'une famille allemande installée à Paris ${ }^{4}$ dans le but, d'après Georges, de l'empêcher de montrer et vendre cette marchandise.

\footnotetext{
${ }^{1}$ Minutes de Léomont, LXXXVII, au Minutier Central des Archives Nationales.

2 Revue d'histoire de l'Amérique française, X, 3: 336.

3 Vente du 8 novembre 1603 et demande de mainlevée du même jour, LVII, 13: fo. 543 et idem: fo. 544.

${ }^{4}$ Nouveaux Documents sur Champlain et son époque, Publication des Archives du Canada (Ottawa, 1967), 151, note 2. Actes du 92e congrès des Sociétés savantes, Section d'Histoire moderne et contemporaine (1967), II : 9-23.
} 
Pour parer à toute éventualité, le marchand rochelais, logé rue de La Harpe, vendit le lot saisi, le 8 novembre 1603, à deux maîtres chapeliers parisiens, François Pérot, demeurant rue de la Vieille-Draperie, et Jacques Lefébure, demeurant rue Barillerie, paroisse Saint-Barthélémy. Il s'agissait de deux assortiments de peaux de castors de bonne qualité et formant un lot avec deux autres assortiments déjà vendus et livrés par Georges aux mêmes clients depuis quelques jours. Les chapeliers déclarèrent avoir vu les castors saisis, les avoir trouvés bons et accepter de se joindre à toute instance engagée par Georges devant les juges consuls de Paris ou ailleurs pour obtenir la restitution des peaux, après laquelle Georges s'engageait à livrer immédiatement, en conservant à sa charge les frais de justice dont il devait avancer le montant. Le prix était de 4 livres 10 sous pour chaque castor pris à la douane payable moitié un mois après la livraison et l'autre moitié deux mois après.

Georges se transporta le même jour avec le notaire Janot rue de La Cordonnerie, chez honorable homme Gracine Leroux, marchand pelletier, garde et maître de la pelleterie de Paris et lui signifia qu'il était venu dans la capitale pour obtenir la restitution de ses peaux. Celles-ci étaient pareilles à d'autres que Honton avait vendues et livrées depuis la saisie à cinq mâ̂tres chapeliers parisiens. Georges, qui venait de vendre les siennes, déclara qu'il s'exposait, s'il ne les livrait pas, à des dommages et intérêts dont il rendait les maîtres gardes de la pelleterie responsables ainsi que de ses frais de séjour. Il était, en conséquence, prêt à se pourvoir s'il n'obtenait satisfaction.

Leroux répondit simplement qu'il en ferait part à ses compagnons gardes, et le séjour de Georges à Paris se prolongea au moins jusqu'au 9 décembre suivant. Il tomba alors d'accord avec ses acheteurs pour résilier la vente de castors, ceci "pour certaines considérations à ce les mouvans", motifs sibyllins ne permettant pas d'en savoir davantage. ${ }^{5}$

Ces premiers documents sont beaucoup moins explicites que la cession effectuée par de Mons, le 10 février 1604, à Jean Macain et Samuel Georges du tiers des peaux de castors et de loutres provenant du Canada et d'Acadie devant lui revenir ainsi qu'à ses associés rouennais, pendant deux ans, contrat compliqué, mais aussi très minutieux et détaillé. L'unité marchande, "la peau", serait facilement intelligible si son estimation était différente selon la qualité, mais l'acte fixe le prix d'une peau, puis

${ }^{5}$ Annexe à la vente du 8 novembre 1603 , déjà citée. 
énumère des qualités qui sont estimées quantitativement par rapport aux peaux étalon. Celles-ci sont les grandes peaux neuves marchandes, crues ou apprêtées en robes ou autrement, valant une peau chacune et 4 livres 7 sous 6 deniers tournois livrées à Saint-Malo ou à Rouen. Les peaux faites avec le bas du poil sont estimées seulement à la valeur de deux peaux pour trois; une troisième catégorie non précisée ne vaut qu'une peau pour deux; enfin, les peaux issues de petits animaux ne valent qu'une peau pour trois. Toutes les peaux dont il vient d'être question sont réputées marchandise neutre, c'est-à-dire vraisemblablement neuve par suite d'une erreur du scribe. ${ }^{6}$ Le contrat n'indique pas expressément la valeur des vieilles peaux donnant d'abord une solution pour une difficulté particulière: les robes moitié neuves, moitié vieilles ou en morceaux sont réputées marchandise vieille. Les robes de huit peaux comptent pour sept peaux, celles de sept pour six, celles de six pour cinq, celles de cinq pour quatre, celles de quatre pour trois, celles de trois pour deux et celles de deux pour une. Il semble que les vieilles peaux et les morceaux soient réévalués proportionnellement avec le nombre de peaux neuves figurant dans la livraison. La vieille peau qui ne compte pas si elle se trouve avec une seule peau neuve subit seulement une diminution d'un quart si elle est accompagnée de quatre peaux neuves. Les peaux isolées, décousues de robes ou non, de procédure compétente, c'est-à-dire vraisemblablement neuves mais de dimensions moindres que les peaux étalon, valent quatre peaux pour cinq. Les peaux tirées des manches ou constituées avec des morceaux valent seulement une peau pour trois. Les peaux étalon livrées à La Rochelle sont payées cinq sous de plus à condition que les marchandises réputées vieilles ne dépassent pas le quart de la livraison, soit le tiers des réputées neuves. Si leur nombre est plus grand, la peau étalon tombe à soixante sous. Le prix devait être payé moitié à la livraison et moitié trois mois plus tard à Rouen dans la maison de Corneille de Bellois où de Mons fit élections de domicile.

L'organisation générale de la Compagnie intervint, car les marchands rochelais avaient le droit de déduire de leur premier paiement ce qu'ils pouvaient avoir avancé pour le compte de Pierre du Gua et de ses associés rouennais pour le fret des navires et les gages des équipages. Ils étaient donc considérés comme pouvant servir de banquiers et le prix convenu devait

${ }^{6}$ Nouveaux Documents, op. cit. - Bulletin philologique et historique (1960): 378. 
être avantageux puisqu'ils bénéficiaient d'une option leur permettant de continuer le marché pendant la durée de la Compagnie en prévenant au mois de janvier, un an d'avance. De ce contrat mal rédigé, d'une interprétation souvent difficile, ayant, quoique passé à Rouen, toutes les caractéristiques des rédactions rochelaises, certains éclaircissements peuvent cependant être déduits sur la valeur des peaux de castor. Pour les deux grands spécialistes de La Rochelle qui paraissent avoir été les acquéreurs les plus importants de la compagnie de Mons, la plus belle peau atteignait, en 1604, au maximum quatre livres sept sous six deniers, soit environ neuf mille deux cents francs 1958, suivant l'estimation proposée par nous ', à condition de livrer à La Rochelle, seulement quatre livres sept sous six deniers, soit environ huit mille sept cent trente francs, si l'acheteur devait faire venir la marchandise de Saint-Malo ou de Rouen. Le prix tombait facilement à soixante sous la peau, soit trois livres et six mille francs 1958 pour les peaux neuves, même à deux livres dix-huit sous et cinq mille huit cents francs lorsqu'elles avaient le poil bas, même à une livre neuf sous, soit deux mille neuf cents francs pour les peaux de petits castors. Les vieilles peaux n'étaient acceptées qu'à contrecœur, sans doute parce qu'elles étaient imposées par les vendeurs. Le système compliqué consistant à faire intervenir leur quantité pour fixer le prix des belles peaux ne peut être que l'effet d'un désir certain d'éviter leur acquisition, en en empêchant l'estimation.

On peut toutefois déduire de l'ensemble que le prix moyen d'une peau de castor en 1604 était d'environ trois livres. L'assimilation des peaux de loutres s'appliquerait pour le commerce des pelleteries sinon pour la fabrication des chapeaux. Il résulte d'une mainlevée, donnée le 21 octobre 1604 par les maîtres pelletiers de Paris, que la peau de castor était une unité marchande pouvant être constituée avec plusieurs peaux soit de castors soit de loutres, car d'après cet acte, des castors et des loutres représentaient la valeur de huit peaux de castors communs. ${ }^{8}$ L'appellation "peaux de castor" prise au sens large ne représentant pas une unité marchande était remplacée par l'abréviation "castors" et ce ne fut pas un fait isolé. Dès le 20 octobre 1607 , Pierre du Gua donna une procuration pour faire saisir les quantités de 1280 et 500 castors ou environ. D'après ce document les 500 castors sont " 500 castors et loutres ët autres peaux", puis "500 castors et loutres". ${ }^{9}$ Une autre procuration

${ }^{7}$ Revue d'histoire de l'Amérique française, XIII, 4: 573.

8 Ibid. (mars 1966), XIX, 4: 504. - Nouveaux Documents, op. cit., 89.

9 Ibid., 506 et 145. 
du même lieutenant général du 20 octobre suivant énonça les "castors, loutres, martres".10 Une mainlevée donnée le dernier novembre 1607 pour le premier lot faisant l'objet de la procuration du 20 octobre 1607 répéta seulement "les castors"."11 Il en fut de même pour le second lot. On peut supposer que la première procuration avait indiqué les loutres par précaution, dans l'incertitude de la composition exacte du lot reconnu ensuite comme ne comprenant que des castors, mais il n'en reste pas moins acquis que l'expression "castors" désignait une certaine quantité de peaux de castors et qu'on continuait d'avoir tendance à assimiler aux peaux de castors celles des loutres et même celles des martres.

Une valeur minima de sept livres et demie pour une peau de castor ou de loutre à La Rochelle en 1607 paraît établie par une déclaration de Samuel Champlain en date du 28 décembre de cette année-là dont il résulte que, d'après ce que lui avait dit Samuel Georges, Ralluau avait, au retour des navires, vendu au marchand rochelais 50 castors et 20 loutres traités par Champlain avec l'autorisation de Pierre du Gua pour sept livres dix sous pièce. Le découvreur, qui avait ratifié par erreur et accepté un acompte pour ne pas désavouer Ralluau, préféra reprendre ses fourrures en apprenant que la vente n'avait pas été réalisée. ${ }^{12}$ Le 6 février 1608 Pierre de Champdoré céda, pour 600 livres tournois, à un secrétaire du roi nommé Jacques Bouchet, 91 castors bons, loyaux et marchands qui se trouvaient à La Rochelle depuis 1606 entre les mains de Samuel Georges, soit pour un prix d'un peu plus de six livres et demie l'unité. Le prix des castors subissait donc une baisse ${ }^{13}$ qui semble avoir continué. La précision "peaux de castors" persista dans la rédaction de curieux contrats de ventes passés devant les notaires parisiens sous la forme de prêts à la grosse aventure et, le 23 décembre 1609, François Gravé vendit aux risques et périls de la mer à Mathieu Duisterlo cent peaux neuves bonnes, loyales et marchandes pour 772 livres, ce qui donne 7 livres 14 sous l'unité, 5 livres 16 sous si on estime les risques du prêt à la grosse aventure à $25 \%$ et seulement 5 livres 7 sous si on estime ces risques à $30 \%$ comme en Normandie. Le prix était applicable à des marchandises livrées à Honfleur ou à un autre port de décharge du navire transporteur, imprécision permettant de

10 Nouveaux Documents, op. cit., 144.

11 Ibid., 149.

12 Minutes de Cuvillier, XV, XVII, Communication de Mme Jurgens, conservateur des Archives d'Ottawa.

13 Ibid., XXIV : 232. 
penser qu'il s'agissait d'un prix moyen. ${ }^{14}$ Mais, le 4 octobre 1610 , sept chapeliers parisiens promirent à Jean de Biencourt un prix de 7 livres 10 sous pour une marchandise livrée à Dieppe. Les peaux devaient être neuves, bonnes, loyales et marchandes, formule qui semblait prendre corps, mais on retrouve le système compliqué de la peau étalon. Les peaux basses et plates, c'est-àdire "les muées grandes et de recepte" comptaient deux pour une. Les autres moyennes étaient estimées à portion égale, les robes de six à cinq, celles de cinq à quatre, celles de quatre à trois et ainsi conséquemment. ${ }^{15}$ L'interprétation de cet acte important comporte quelques difficultés. On y trouve d'abord "les pelleteries de castor", ce qui ne permet guère de discriminer si les fourrures de l'animal ainsi dénommé sont indiquées au sens large ou au sens restreint, puis "pelleteries et castors", castors étant ici pris au sens restreint. Les loutres sont ensuite assimilées, puis le prix des martres ainsi nettement mises à part reste à fixer avec, cependant, la précision que pour être marchandes elles ne doivent pas être muées.

Les chapeliers avançaient une somme de 12000 livres pour servir à l'achat de marchandises destinées à être troquées contre les "pelleteries et castors", l'expression paraissant donc avoir retenu l'attention du scribe pour être employée dans un sens comportant une discrimination entre les pelleteries et les castors. On peut seulement penser que Poutrincourt pouvait utiliser une partie des avances pour l'acquisition de pelleteries ne servant pas à la fabrication des chapeaux. Le prêt étant consenti sans intérêt, les chapeliers n'avaient pas à supporter les risques du péril de la mer et leurs avances devaient être remboursées sans en tenir compte. Le prix de 7 livres 10 sous doit donc être considéré comme net pour une livraison dans un port de France, ici à Dieppe. Il était même un peu plus élevé, comme augmenté par l'obligation de consentir une avance de 12000 livres bien que les prêteurs aient obtenu ainsi le bénéfice de se voir réserver toute la traite des castors susceptible d'être réalisée par Poutrincourt et ses associés, pouvant, par conséquent, atteindre une valeur dépassant celle des avances. Il faut ajouter qu'une condition particulière pouvait justifier l'élévation $d u$ prix, puisque l'exécution du contrat était subordonnée à l'obtention par Poutrincourt de l'interdiction qu'il demandait alors devant le Conseil privé pour quiconque de négocier avec les sauvages sans sa permission; la durée prévue pour le contrat était celle de ce

14 Nouveaux Documents, op. cit., 200.

15 Ibid., 208. 
privilège. Comme le texte rappelait que Poutrincourt résidait au Port-Royal d'Acadie pour propager le catholicisme, on ne peut éviter de comparer ces indications d'acte notarié avec celles qui ont été données par l'Histoire de la Nouvelle-France de Lescarbot. D'après cet avocat, le prix d'achat d'un castor aux sauvages était monté, depuis 1602, de deux gâteaux ou de deux couteaux à quinze ou vingt. Cependant, durant cette année 1610, des marchands avaient été jusqu'à distribuer gratuitement tout leur stock aux Indiens pour empêcher la sainte entreprise de Poutrincourt. 16

Il n'apparaît pas, en tout cas, que, depuis la suppression du privilège de Pierre du Gua, le prix des castors aît doublé comme l'a prétendu Lescarbot, puisque l'accord éventuel passé avec Poutrincourt dans l'hypothèse de la survenance d'une situation défavorable pour les marchands ne révèle qu'une hausse d'environ $40 \%$ entre 1607 et 1610. Dans l'acte de constitution de la Compagnie du Canada du 15 novembre 1613, les peaux marchandes appartenant à des tiers firent l'objet d'une offre de seulement 3 livres pour éviter la confiscation au port de retour. Il apparaît qu'une certaine surproduction était alors possible car le prix de vente pour les marchandises des associés ne fut pas fixé, faisant l'objet de fortes préoccupations. ${ }^{17}$

Une vente de peaux de castors fut consentie au poids, le 23 septembre 1618 par Etienne Moreau, marchand de La Rochelle, au profit d'honorable homme Nicolas Collin, pelletier et valet de chambre ordinaire du roi, demeurant rue Saint-Denis, paroisse Saint-Nicolas. La marchandise fut très mal désignée quantitativement comme "quatre à cinq cens poisant ou plus sy faire se peult de castors vieils". L'unité marchande était donc devenue la livre pesant de peaux de castors en remplacement de l'unité constituée par la peau étalon. La qualité des peaux continua, cependant, d'être déterminée car la quantité de vieilles peaux faisant l'objet de la vente devait par suite d'une assez étrange conception comprendre un quart de peaux plates et neuves. L'acte précise que ce mélange comprenant des vieilles peaux pour les trois quarts était vendu à un pelletier comme bien loyal et marchand, expression paraissant adoptée; mais nous ignorons combien la livre pesant constituée dans ces conditions représentait de peaux loyales et marchandes..$^{18}$ Quoi qu'il en fût, la livre de 593.

${ }^{16} \mathrm{M}$. Lescarbot, Histoire de la Nouvelle-France (édition Tross), III:

\footnotetext{
17 Nouveaux Documents, op. cit., 310 ss. Extrait de l'Anuario de Estudios Americanos de Séville, XIV : 23.

18 Minutes de Gerbault, II: 94, aux Archives Nationales.
} 
castor au poids de Paris coûta cent quinze sous, donc pas tout à fait six livres.

Mathieu Duisterlo ${ }^{19}$ vendit aussi au poids, le 29 janvier 1621, à honorable homme Claude Bodin, marchand chapelier demeurant sur le pont Notre-Dame, paroisse Saint-Jacques-dela-Boucherie, quatre balles de marchandises de castor pesant ensemble avec les serpillières 1051 livres pour 7256 livres tournois. Ce prix semble indiquer qu'il s'agissait de peaux loyales et marchandes avec une certaine stabilité de valeur pour une telle marchandise entre six et sept livres tournois la livre pesant, sans qu'il soit possible de préciser davantage, le poids des emballages étant ignoré. ${ }^{20} \mathrm{La}$ vente à la peau n'était cependant pas abandonnée car, en 1621, 2500 castors furent vendus 15011 livres, soit près de six livres pièce. ${ }^{21}$ Le rapprochement de ces opérations donne à penser qu'une livre pesant de castor représentait à peu près une peau loyale et marchande. Le Conseil privé estima aussi les castors à la peau par arrêt du ler avril 1622, en imposant à la seconde compagnie de Montmorency de reprendre douze castors à l'équipage de chaque bateau servant à la pêche au Canada, si les fourrures avaient été obtenues par don ou échange. Le prix de trois livres tournois pièce paraît avoir été fort avantageux pour les Associés, bien que semblant s'appliquer à des cessions réalisées au Canada. ${ }^{22}$ Dolu, Duisterlo, Porée et Boyer convinrent en effet, le 20 décembre 1622, que les castors venant de la Nouvelle-France seraient vendus cent quatre sous, soit un peu plus de cinq livres tournois la livre pesant en 1623, 1624 et $1625 . .^{23}$ Le commerce du castor avait alors pris une extension considérable, car, à cette époque, Boyer fit peser 6021 livres de castors représentant donc environ 40000 livres tournois et 80 millions de francs 1958 , d'après notre estimation. ${ }^{24} \mathrm{La}$ méthode consistant à stipuler un prix de rachat avantageux pour les compagnies privilégiées s'amplifia encore avec la Compagnie des Cent-Associés, tandis que le marché principal se trouvait fixé à Paris. Le huitième article des privilèges qui furent accordés par le roi le 7 mai 1627 porte que les Français habitués avec leurs familles et ne dépendant pas de la Compagnie pourraient traiter librement les pelleteries avec les sauvages, mais à condi-

19 Sur lui, Actes du 92e congrès des Sociétés savantes (1967), déjà cités. 20 Minutes de Journel, XXXV : 83, aux Archives Nationales.

21 Arrêt du Conseil privé du 3 avril 1626 au profit de Guillaume de Caen, V $V^{6}$ 9, pièce 13, aux Archives Nationales.

$22 \mathrm{~V}^{6} 214$, ibid.

23 Arrêt du 3 avril 1626, déjà cité.

24 Revue d'histoire de l'Amérique française, XIII, 4: 573. 
tion de remettre les castors à la Compagnie tenue de les accepter sur le pied de 40 sous, vraisemblablement au Canada. ${ }^{25}$

Les directeurs de la Compagnie décidèrent, le 21 septembre 1631, que les castors qui avaient été livrés en application de cet article 8 seraient remis à Jean Tuffet pour en tenir compte et, le 22 octobre 1631, que ceux qui avaient été envoyés directement à Tuffet par les gens de Charles Turgis, dit de La Tour, pour les vendre, seraient retenus au même prix. Il s'agissait de castors remis par Vincent Jorré au capitaine Bernard Marot ${ }^{26}$ suivant reçu du 10 octobre 1630 et d'autres remis par le même Jorré et un certain Thomas Laniel à Laurent Ferchault, maître du navire Le Petit-Saint-Jean à la même époque. Jorré avait remis pour sa part vingt-et-un castors et demi qui furent remis à Tuffet avant le 23 juillet 1631. Cependant, l'application de l'article 8 ne fut pas admise en définitive pour cette espèce à la suite de longs procès, pour des raisons d'équité qui semblaient naturelles, puisque Charles Turgis ${ }^{27}$ et ses droits en Acadie avaient été complètement négligés lors de la constitution de la Compagnie des Cent-Associés. Une sentence du présidial de La Rochelle du 16 avril 1638 condamna la Compagnie à payer à Jorré la valeur de 123 castors $1 / 2$ au total, en tenant compte du temps de l'envoi, c'est-à-dire de l'époque de la livraison au dire de personnes compétentes dont Jorré devait convenir avec Tuffet. Cette décision fut confirmée par un arrêt du Conseil privé du 5 mars 1641 nous renseignant sur cette affaire ${ }^{28}$, qui accorda en plus à Jorré les intérêts de sa créance à partir du jour de sa demande avec la faculté de faire nommer un expert par le rapporteur de l'instance devant le présidial de La Rochelle à défaut d'exécution d'une désignation déjà faite. Jorré obtint en définitive, après transaction, une créance de 2253 livres dont il faut déduire les intérêts avant de pouvoir rechercher la valeur qui fut accordée pour une peau. Celle-ci doit être située entre 18 livres au maximum et 12 livres au minimum. D'après la déclaration faite par Nicolas Goubert, marchand bourgeois de Dieppe, le 12 juillet 1631, devant les notaires de cette ville, un castor partant de la main des sauvages pesait une livre et demie. ${ }^{29}$ Une certaine incer-

25 "La Compagnie de la Nouvelle-France et la restitution de l'Acadie", Revue d'histoire des colonies (1955), XLII: 75.

26 Ibid.: 77.

27 "Du nouveau sur les La Tour", Revue généalogique canadiennefrançaise, XI: 21.

28 V6 164, aux Archives Nationales. fo. 143 .

29 Arrêt du Conseil d'Etat, du 27 août 1634, Fonds français 16738, 
titude subsiste donc pour le poids de la peau de castor au cours des opérations commerciales successives, mais Jorré fut, en tout cas, payé sur la base d'au moins huit livres tournois la livre pesant et plus vraisemblablement un peu mieux.

Il faut dire que la guerre puis la perte de Québec avaient causé une hausse considérable des prix. Le 11 septembre 1631, André Le Coultre et Jean Maillard, le premier, pelletier, et le second, chapelier, exerçant tous deux à Paris, attestèrent devant un notaire de la capitale malheureusement non identifié qu'ils avaient, durant les années 1628, 1629 et 1630, toujours payé la livre de castor en peau 14 et 15 livres tournois. ${ }^{30}$ Bien que, dès 1630, la Compagnie des Cent-Associés ait exercé une action directe en Acadie, différente de celle de Charles Turgis, dit de La Tour ${ }^{31}$, qui avait lui aussi continué de fournir des castors ainsi que les pêcheurs basques agissant en fraude, des achats avaient été faits aux Anglais et même aux Hollandais, notamment par Mathieu Duisterlo qui s'était trouvé en conflit avec un chapelier parisien nommé Pierre Jouvin. Ce dernier passait pour avoir effectué des achats se montant à 100000 livres tournois en Angleterre. ${ }^{32}$ Les prix diminuèrent en tout cas à partir de 1633. D'après une indication malheureusement imprécise, la Compagnie de la Nouvelle-France accepta, cette année-là, une baisse de 40 sous par livre pour les peaux rapportées sur ses bâtiments. ${ }^{33}$ Elle aurait eu alors parmi ses acquéreurs un de ses membres, le mercier parisien Nicolas Libert, mais les renseignements sur ses ventes de castors manquent jusqu'en 1634, année pendant laquelle on trouve la trace d'une organisation concurrente de celle de Mathieu Duisterlo passant pour avoir, avec l'aide d'une activité considérable et même internationale, réussi à constituer un trust des ventes de castors aux chapeliers parisiens. ${ }^{34}$

Dès l'année 1634, un certain nombre des Cent-Associés comprenant Robert Godefroy, conseiller aux conseils du roi figurant au numéro 53 de la liste rectifiée et complétée par nous,

${ }^{30}$ Ibid., fo. 143, vo.

31 "La Compagnie de la Nouvelle-France et la restitution de l'Acadie", Revue d'histoire des colonies (1955), XLII : 79.

32 Très humbles remonstrances à nos seigneurs de parlement par Nicolas Libert, marchand mercier, bourgeois de Paris, et l'un des Associés et Directeurs de la Compagnie de la Nouvelle-France sur la poursuite contre lui intentée par les Maistres chapeliers de la ville de Paris, $4^{\circ} \mathrm{Fm} 19503$ à la Bibliothèque Nationale.

33 Ibid.

34 Ibid. 
Claude de Bragelogne, intendant et commissaire général des vivres (no 54), demeurant tous deux rue de Berry, paroisse Saint-Nicolas-des-Champs, Pierre Desportes, sieur de Lignères (no 74), demeurant rue de La Verrerie, paroisse Saint-Jean, Claude Margonne, receveur général des Finances en la généralité de Soissons (no 56), demeurant rue du Parc-du-Roi, paroisse Saint-Gervais, s'entendirent pour l'achat des castors avec Pierre Robineau, trésorier général de la cavalerie légère de France (no 42), demeurant rue de Berry, paroisse Saint-Nicolas-desChamps, qui rendit des comptes pour les années 1634 et 1635 , semblant ainsi avoir joué le rôle le plus actif ${ }^{35}$, réserve faite de celui de Nicolas Libert (no 104), demeurant rue des Cinq-Diamants, paroisse Saint-Jacques-de-la-Boucherie. Les affaires furent importantes dès la fin de 1634, car les douze bâtiments envoyés par les Cent-Associés en Nouvelle-France revinrent en Bretagne, en Normandie et à La Rochelle. ${ }^{36}$ Le 14 octobre, Libert participa à un achat fait en société particulière avec Charles Daniel, Pierre Desportes et Nicolas Delaistre à Charles Turgis en Acadie. ${ }^{37}$ Le 11 décembre, il passa un accord pour la vente de tous les chapeaux de castor qu'il pourrait fournir ${ }^{38}$ et, bien que les prix des peaux eussent diminué d'un tiers ${ }^{39}$, il participa aux achats de la Compagnie de la Nouvelle-France en 1635 avec Catherine Mortier, veuve de Pierre du Ryer, secrétaire du Roi (no 64), demeurant rue du Faubourg, paroisse Saint-Gervais. ${ }^{40}$

Les associés de la Compagnie de la Nouvelle-France, acquéreurs de castors, passèrent par l'intermédiaire de Robineau, le 27 septembre 1635, à Saint-Pierre-au-Port, village situé à une cinquantaine de kilomètres du Havre, dans l'arrondissement d'Yvetot, un traité ratifié par eux le 22 octobre suivant devant le notaire parisien Mottelet ${ }^{\mathbf{4 1}}$ pour tous les castors provenant de la Nouvelle-France en 1635, 1636 et 1637. L'affaire fut conclue avec Jacques Berruyer, sieur de Manselmont (no 16), Antoine Cheffault (no 62) et Jean Rozée (no 36), alors préposés pour les affaires et embarquements de la Compagnie générale de la Nouvelle-France et de la Compagnie particulière, c'est-à-dire $\mathrm{LI}: 262$.

35 Inventaire des biens de Pierre Desportes, du 12 août 1645, Richer,

${ }^{36}$ Recueil des Gazettes de Renaudot (1634), 575, $4^{\circ} \mathrm{Lc}^{21}$ à la Bibliothèque Nationale.

37 Transaction du 11 janvier 1639, XXXV: 157.

38 LX: 5.

39 Recueil des Gazettes, déjà cité.

40 Inventaire des biens de Pierre Desportes, déjà cité.

41 Transaction du 7 février 1637, minutes de Rémond, XVI: 74, d'après un acte perdu, ne figurant pas dans XC : 198 . 
la Compagnie du fleuve Saint-Laurent, mais une difficulté survint avec les préposés de cette dernière compagnie qui fut portée devant un Conseil du Roi et Robineau demanda, le 15 janvier 1637 , la résolution du traité à la Compagnie générale. Celle-ci commença par élire, pour conduire ses affaires pendant les années 1637 et 1638, six nouveaux directeurs: Isaac Martin de Mauvoy (no 3), demeurant rue des Mauvais-Garçons, paroisse SaintJean, Robert Godefroy que nous connaissons comme acquéreur de castors, Charles de Boisseret connu comme ayant joué un rôle important à La Guadeloupe (no 148), Charles Fleuriau (no 51), Jean Verdier, secrétaire du roi (no 59), demeurant au Petit-Cloître-Saint-Jacques-de-l'Hôpital, paroisse Saint-Eustache et d'après le document, Mousnier ${ }^{42}$, c'est-à-dire vraisemblablement Louis Musnier, marchand de Rouen (no 155). Martin de Mauvoy, premier directeur de la Compagnie particulière fut désigné avec Antoine Cheffault, sieur de La Renardière, demeurant au Petit-Cloître-Saint-Jacques-de-l'Hôpital, paroisse SaintEustache, ainsi que deux associés de la Compagnie générale, Gabriel Lattaignant, ancien major de la ville de Calais, demeurant rue du Monceau, paroisse Saint-Germain, pour rechercher un accord. Ils décidèrent, le 7 février 1637, que le traité serait exécuté pour l'année 1635 et que les acquéreurs régleraient à Berruyer, Cheffault et Rozée ès qualités, avant le dernier février, un solde de 14079 livres 18 sous restant à payer sur leur achat se montant à 284500 livres lors du retour de la flotte en 1635, suivant une promesse donnée par Robineau le 7 novembre de la même année. La même solution fut acceptée pour les deux autres années 1636 et 1637, moyennant 15000 livres dont 2000 furent payées comptant en pistoles d'Espagne. Desportes et ses associés s'engagèrent à régler le solde de 13000 livres à Dieppe dès la première réquisition de Cheffault et en fourrures de castors, ce qui nous intéresse particulièrement. Il s'agissait en effet de castors assortis, gras pour les deux tiers, maigres pour un tiers, valant 10 livres la livre pesant au poids de la vicomté de Dieppe avec la précision que les 13000 livres tournois étaient bien représentées par 1300 livres pesant. Cette marchandise provenant des retours de 1635 avait fait l'objet d'une saisie pratiquée à la requête des consorts Cheffault qui devaient en donner mainlevée.

Nous ignorons ce que les préposés firent des castors rapportés en 1636 qui restaient à leur disposition à Dieppe, mais cer-

42 Expédition signée Lamy de la délibération de la Compagnie de la Nouvelle-France du 15 janvier 1637 jointe à la transaction déjà citée du 7 février suivant. 
tains associés continuèrent en tout cas le commerce personnel des fourrures de castor. Le 11 janvier 1639, Guillaume Desjardins, intendant de Charles Turgis qualifié ici Monsieur de La Tour, accepta de payer à Charles Daniel, Nicolas Libert, Pierre Desportes et Nicolas Delaistre 4800 livres pour 400 peaux de castors, loutres et orignaux, soit 12 livres la peau, la valeur de celles d'orignaux n'étant malheureusement pas précisée. ${ }^{43}$ Le 13 janvier 1639, Charles Daniel céda au prix de 8 livres 5 sous la livre pesant à Nicolas Libert tous les castors en peaux et poils lui appartenant à Dieppe entre les mains de Berruyer, Cheffault et Rozée. ${ }^{44}$ Le 10 octobre 1639, Guillaume Martin, sieur de La Vernade (no 70), demeurant rue et cousture Sainte-Catherine, paroisse Saint-Paul, agissant comme procureur de noble homme Louis Sanguin, sieur de Regron, receveur général des Finances en Bretagne (no 158) et d'Antoine Chevalier, écuyer, sieur de Surgibault (no 159), associés chacun pour une part dans la Compagnie particulière de la Nouvelle-France, vendit 522 livres pesant de castors qui appartenaient à Guillaume Martin et à Sanguin pour 8 livres tournois la livre pesant à Charles Baussay, maître chapelier à Paris qui en céda la moitié à Robert Regnault, aussi maître chapelier et associé de la Compagnie générale (no 140) particulièrement actif. ${ }^{45}$

Une baisse importante pouvait avoir alors commencé, car en 1640 Jacques Le Boeuf remit à Nicolas Denys trois paquets de 20 castors valant 60 livres et pesant 92 livres. Le document indique que "le castor" signifiait "la peau de castor". Celle-ci pesant donc environ 1 livre et demie conservait des caractéristiques de peau étalon car une barrique de 65 castors comprenait deux petits paquets de 40 "kichipan" pesant 16 livres comprises dans les 108 livres, poids total du contenu de la barrique.

La peau de castor pesant donc environ 1 livre et demie valait par conséquent 3 livres tournois et la livre pesant de castor environ 2 livres. La livraison comprit un paquet de trente demicastors, cette expression semblant ici signifier qu'il s'agissait de moitiés de peaux. ${ }^{46}$

Les variations de prix pouvaient être importantes et rapides, sauf différences dans les qualités que le système des peaux étalon semble devoir faire écarter, car le 19 octobre 1645 le capitaine Sauvic vendit à Emmanuel Le Borgne 33 peaux de castors et 15

46 B 5056 aux Archives de la Charente-Maritime à La Rochelle.

43 XXXV: 157.

44 XXX: 155.

45 LII : 15. 
peaux de loutres à 10 livres tournois pièce. ${ }^{47}$ Les peaux d'orignaux dont nous savons peu de chose servaient en tout cas à faire des buffles dont la façon coûtait 6 livres à Bayonne le 28 avril 1626.48 Une peau de loup-cervier fut estimée le 20 novembre 1625 seulement 60 sous avec un petit morceau de luberne. ${ }^{49}$

Voilà donc quelques modalités de ce commerce compliqué des fourrures entre l'Amérique et la France au début du XVII siècle.

47 Registre de Teuleron de 1645 , idem.

48 Minutes de Pierre Harran III E 3598 aux Archives des PyrénéesAtlantiques.

49 Inventaire d'Antoine Féras, LXXXVII : 110. 